\title{
Are Microsatellite Patterns Specific for Tumor Types? A Pilot Investigation
}

\author{
Tiffany Haiduk ${ }^{1}$, Michael Brockmann ${ }^{1}$, Christoph Schmitt ${ }^{2}$, Ramona-Liza Tillmann ${ }^{1}$, \\ Monika Pieper ${ }^{1}$, Jessica Lüsebrink ${ }^{1}$, Oliver Schildgen ${ }^{1, * \mathbb{D}}$ and Verena Schildgen ${ }^{1, *}$ \\ 1 Kliniken der Stadt Köln, Klinikum der Privaten Universität Witten, Ostmerheimer Str. 200, \\ 51109 Köln (Cologne), Germany; tiffanyhaiduk@hotmail.com (T.H.); brockmannm@kliniken-koeln.de (M.B.); \\ tillmannr@kliniken-koeln.de (R.-L.T.); pieperm@kliniken-koeln.de (M.P.); \\ luesebrinkj@kliniken-koeln.de (J.L.) \\ 2 GENOPATH GbR, Heilsbachstraße 15, 53123 Bonn, Germany; cschmitt@genopath.de \\ * Correspondence: schildgeno@kliniken-koeln.de (O.S.); schildgenv@kliniken-koeln.de (V.S.)
}

Received: 22 July 2020; Accepted: 1 September 2020; Published: 4 September 2020

\begin{abstract}
Microsatellite testing is an emerging field of molecular pathology, as microsatellite instability (MSI) appears to be a predictive biomarker for some cancers. Although multiple studies on microsatellites have been published, recent observations suggest that the microsatellites that define instability differ between tumor entities. This assumption is confirmed by the present study that compared different MSI assays validated for colorectal cancer. Whilst all assays deliver the same MSI/MSS status for colorectal cancers, they differ for tonsillar tumors, leading to the hypothesis that MSI patterns are tumor-type specific.
\end{abstract}

Keywords: microsatellite instability; MSI; colorectal cancer; tonsillar tumors; MSI pattern

\section{Introduction}

Microsatellites (MS) are repetitive sequences in the genome that are present throughout the genome. These repeat sequences are more commonly found in non-coding regions on many alleles, but can also be found in coding regions. The mismatch repair system (MMR) is responsible for the correct detection of microsatellites in DNA replication. Due to the high repetitiveness of these sequences, slippage can occur causing a shift in the genomic sequence. This is detected by the MMR and corrected. However, if the MMR is defective, the mutation rate increases and the slippages causing insertions and or deletions go undetected, thereby leading to tumorigenesis.

The defective MMR leads to altered microsatellite replication, also called microsatellite instability (MSI). Up to 70-95\% of sporadic colorectal cancers (CRC's) have high microsatellite instability, defined by $40 \%$ instable markers (two out of five) if applying the Bethesda panel for diagnostics [1]. Knowing the MS status of tumors allows for a better prognosis of overall patient survival and treatment possibilities [2]. It is therefore recommended to determine MS status before treatment to give proper prognosis and treatment to the patient [3].

Beyond the Bethesda panel, other testing systems are available on the market, such as the Biocartis Idylla platform, or the MSI sequencing assays from Promega. Thereby, Biocartis uses seven novel MS markers (ACVR2A, BTBD7, DIDO1, MRE11, RYR3, SEC31A, and SULF2) for detecting instability in colorectal tumors. This system aims at determining MS status without paired normal tissue, as the more common method of MSI detection is PCR amplification of normal and tumor tissue to be compared to detect instability. For MSI status determination, the Bethesda panel consisting of five microsatellite markers (BAT25, BAT26, D5S346, D2S123, and D17S250) has been previously used for uniform MSI status determination [1]. Biocartis recommends use of this product for colorectal solid 
tumors. The Promega MSI analysis system also uses a different set of target microsatellites, namely NR-21, BAT-25, BAT26, NR-24, MONO-27, Penta C, and Penta D and is manufacturer-approved for the detection of MSI in colorectal cancers.

In 2018, we published a de-multiplexed method for the detection of MSI based on the Bethesda panel and the QIAxcel Advanced platform (Qiagen, Hilden, Germany) [4], which we successfully used for the analyses of colorectal cancer specimens, but which was also used for the analysis of MSI in tonsillar tumors [5]. As the in-house method, although cheap, requires long hands-on time, we consequently wanted to test the commercially available methods for MSI testing, not least as the Idylla system did not require complex pre-analytical steps.

In this study, however, the test was also used on samples of the tonsil to determine whether MSI could also be detected in this tissue using the given test.

\section{Materials and Methods}

Tumor specimens of tonsillar tumors were formalin fixed paraffin embedded tumor tissues, in which the tumor was visible macroscopically. The usage of those samples was in agreement with a vote from the ethical committee of the University of Witten (vote number 151/2016). One slide was used to determine the boarded of the tumor and the FFPE blocks were subject to macro-dissection, thus the estimated tumor content used for each analysis was $>80 \%$. Samples from colorectal tumors with known MSI/MSS status originated from archived round robin trials the lab has participated in the past three years.

Three methods were used in the study, namely a previously published MSI testing protocol established on the Qiagen QIAxcel Advanced platform, a commercial cassette assay (Idylla, Biocartis, Belgium), and a commercial assay for capillary electrophoresis (Madison, WI, USA), of which the previously published protocol was set as internal gold standard [4].

The Idylla ${ }^{\mathrm{TM}}$ system (Biocartis, Mechelen, Belgium) is a compact and time efficient automated method to detect common pathogenic mutations found predominantly in lung and colorectal cancers. For these tests, either 5-10 $\mu \mathrm{m}$ FFPE slices of tissue sample or liquid samples were used. For solid samples, the slices are placed between two filters moistened with DNase free water, and carefully placed into the Idylla ${ }^{\mathrm{TM}}$ cartridge. With liquid samples, the sample is pipetted directly onto the clear plate at the bottom of the cartridge. This is then inserted into the instrument where liquefaction, cell lysis, nucleic acid extraction, amplification and analysis take place. The analysis takes up to $150 \mathrm{~min}$ depending on the mutation analysis being run.

MSI testing using the Promega assay (MSI Analysis System, Version 1.2) was performed strictly according to the manufacturer's protocol.

\section{Results}

The samples for microsatellite testing were previously tested using PCR amplification and detection using QIAxcel Advanced. Of the 18 samples tested, 10 had unstable microsatellites and 8 samples were stable using QIAxcel Advanced. Idylla ${ }^{\mathrm{TM}}$ however, detected MSI in six samples and stable microsatellites in 12 samples. This is shown in Table 1, as some of the samples that were previously identified as stable using QIAxcel Advanced were detected as unstable using Idylla ${ }^{\mathrm{TM}}$, and vice versa. For this reason, some samples for which residual FFPE specimens were available were tested for MSI using a different assay from Promega (Madison, WI, USA). The results of this additional test are listed in Table 1.

There were many discrepancies in the results between the QIAxcel Advanced and Idylla testing, leading to a suspected lower sensitivity and NPV at $60 \%$ and $66.66 \%$ respectively. Both the specificity and PPV, however, were $100 \%$ using the Idylla ${ }^{\mathrm{TM}}$ test. All samples tested with the Promega assay were MSS, except sample 7, where there was not enough DNA for the analysis. 
Table 1. MSI results of samples tested using QIAxcel Advanced PCR amplification and Idylla ${ }^{\mathrm{TM}}$. Results of samples sent for external testing using Promega kit also listed. In some cases there was not enough residual FFPE specimen left for Promega testing therefore no testing (n.t.) was possible.

\begin{tabular}{llllc}
\hline Sample & Tissue Source & QIAxcel Advanced & Idylla & Promega \\
\hline MSI 01 & Colon & MSS & MSS & n.t. \\
\hline MSI 02 & Colon & MSI & MSI & n.t. \\
\hline MSI 03 & Colon & MSS & MSI & n.t. \\
\hline MSI 04 & Colon & MSI & MSI & n.t. \\
\hline MSI 05 & Colon & MSS & MSI & n.t. \\
\hline MSI 06 & Colon & MSI & MSS & DNA concentration too \\
\hline MSI 07 & Tonsil & MSI & MSter extraction \\
\hline MSI 08 & Colon & MSI & n.t. \\
\hline MSI 09 & Tonsil & MSI & MSI & n.t. \\
\hline MSI 10 & Colon & MSI & MSS \\
\hline MSI 11 & Colon & MSI & MSS & MSS \\
\hline MSI 12 & Colon & MSI & MSS & MSS \\
\hline MSI 13 & Colon & MSS & MSS & n.t. \\
\hline MSI 14 & Colon & MSS & MSS & n.t. \\
\hline MSI 15 & Colon & MSS & MSS & n.t. \\
\hline MSI 16 & Colon & MSS & n.t. \\
\hline MSI 17 & Colon & MSS & MSS \\
\hline MSI 18 & Tonsil & MSS & MS & MS \\
\hline
\end{tabular}

\section{Discussion}

Microsatellites are small repetitive sequences spread throughout the genome, present in both coding and non-coding regions [2]. Due to the repetitiveness of these sequences, the DNA polymerase is prone to slippage when synthesizing these microsatellite sequences. The error in the DNA, however, is detected and repaired by the DNA mismatch repair (MMR) system [6]. This, however, is not possible if the MMR system is defective, which causes instability in the microsatellites. The instability can in turn cause frameshift mutations in the genome, leading to disrupted protein function. This deactivation of the MMR genes can be sporadic, as it is in many CRC cases, or caused by a germline mutation, causing Lynch syndrome [7]. In many non-hereditary CRC cases, it has also been detected that MSI is not in fact caused by mutations of the MMR genes, but rather hypermethylation of the MMR genes, specifically MLH1, thereby implying demethylation as a method to restore functionality of these genes [8]. Knowing whether MSI is caused by spontaneous deactivation or mutations in the MMR genes, or whether it is inherited, can lead to the detection of Lynch syndrome and differential treatment.

There have been many studies showing the presence of microsatellites (MS) in multiple cancers [9]. The study from Bonneville in particular showed the presence of microsatellite instability in 27 types of cancers. Microsatellite instability, however, is yet only recommended to be tested in endometrial and colorectal cancers [9]. The European Society for Medical Oncology (ESMO) recommends testing for MSI in colorectal patients, even though it is present in 4-8\% of metastatic CRC tumors [3]. Even though the percentage of patients affected with MSI is low, it is still of importance, as patients with MSI tumors tend to have a better prognosis than those with stable microsatellites [7]. Patients with CRC and MSI have a better response to immune checkpoint therapy, making the testing for MSI an important criteria, 
not only to offer the patient the most useful treatment, but also useful for the development of targeted treatments [9].

The development of accurate and fast testing is therefore of importance to many biotechnology companies. The current standard for MS analysis is either using immunohistochemistry (IHC) or PCR analysis and subsequent visualization using electrophoresis.

IHC measures the expression of the MMR proteins MLH1, MSH2, PMS2, and MSH6. If MSI is present, there would be no function in one or multiple of the MMR genes [9]. IHC testing, however, is not sensitive enough as it does not detect all mutations present in these genes that could lead to MSI [2]. However, for the samples used in this study, no IHC testing was available, and as for the round robin samples, solely molecular information was provided, while for tonsil samples, such testing was not performed as limited sample volume/slides were available for study purposes. Thus, future studies should also address the correlation if IHC and molecular markers more in depth.

For PCR analysis, the Bethesda panel is often used. This panel was created to standardize MSI testing using five markers, two mononucleotide markers (BAT25 and BAT 26) and three dinucleotide markers (D5S346, D2S123, and D17S250). This panel, however, is limited in its ability to correctly classify results. This panel differentiates between high MSI (MSI-H), low MSI (MSI-L), and stable microsatellites. The mononucleotide markers are more sensitive and specific, whereas the dinucleotide markers are more prone to mutation, leading to false results [2].

Knowing this, the samples in Cologne were originally tested using four of the five markers from the Bethesda panel and six additional markers. The following markers were used to analyze samples: BAT25, BAT26, APC, D17s250, D2s123, D13s153, BAT40, Mycl1, D18s58, and D10s197 [10], where D5s346 from the Bethesda panel is replaced with APC. These samples were tested in conjunction with their normal tissue samples and the results compared to detect instability in the listed markers. This panel was used for both tonsil and colorectal samples and showed instability in both tissue types. The ability of this panel to detect instability in different tissue types is very important, as microsatellite instability has been linked to tumor and tissue specificity, as certain genes are more susceptible to MSI frameshifts than others [7].

The Bethesda panel or extended versions thereof, have a distinction between highly unstable microsatellites and low unstable microsatellites. Using both PCR with subsequent electrophoresis and Idylla $^{\mathrm{TM}}$ testing, no distinction was made between samples being MSI-L or MSI-H.

The Idylla ${ }^{\mathrm{TM}}$ test has been specifically developed to be used on colorectal tissue with seven biomarkers targeting detection of MSI in CRC: ACVR2A, BTBD7, DIDO1, MRE11, RYR3, SEC31A, and SULF2. The main advantage of the Idylla ${ }^{\mathrm{TM}}$ system is that it does not require matching normal tissue samples for microsatellite status determination. During our testing Idylla ${ }^{\mathrm{TM}}$ had a sensitivity of $60 \%$. This means the test can correctly detect MSI in $60 \%$ of the samples. The test has a specificity of $100 \%$, meaning that all the samples that were stable using the QIAxcel Advanced test method were also stable using Idylla ${ }^{\mathrm{TM}}$. With a PPV of $100 \%$, one can be certain that if a positive result for MSI was given using Idylla ${ }^{\mathrm{TM}}$, the result is in fact correct. The NPV, however, is lower at $66.66 \%$. That means, if the test result comes back as MSS, there is a $33.34 \%$ chance that this result is not correct.

As mentioned before the Idylla ${ }^{\mathrm{TM}}$ test was also used on tonsil samples. Of the 18 samples tested, three of them were tonsil samples. All three of these samples were incorrectly classified. Samples 7 , 9, and 18 were all tonsil samples, originally unstable in at least one biomarker tested using QIAxcel Advanced. Idylla ${ }^{\mathrm{TM}}$ classified all three samples as MSS, meaning there is no instability detected in the seven biomarkers from Idylla ${ }^{\mathrm{TM}}$, thereby reinforcing the assumption that microsatellites display tumor and environment specificity with the biomarkers present [7]. As the Idylla ${ }^{\mathrm{TM}}$ testing is targeted for colorectal samples, it can be assumed that the biomarkers in this panel are thereby colon specific and are not present or prone to instability in samples from other tumor environments.

Excluding the tonsil samples, the sensitivity and the NPV of the Idylla ${ }^{\mathrm{TM}}$ method both increases by more than $20 \%$. The sensitivity of only the colorectal samples increases to $85.7 \%$ and the NPV increases to $88.8 \%$. Both the specificity and PPV remain the same at $100 \%$. With this in mind, it can be stated that 
the Idylla ${ }^{\mathrm{TM}}$ testing is much better at detecting instability in colorectal samples and is not suitable for use on other tumor samples. Even with testing only colorectal samples, Idylla ${ }^{\mathrm{TM}}$ testing still incorrectly identified three samples. Samples 11 and 12 were both unstable using QIAxcel Advanced, with 2 and 1 marker unstable respectively. The disagreement between both testing methods could be due to lack of neoplastic cells present in the FFPE blocks used. The QIAxcel Advanced testing was done two years prior to the Idylla ${ }^{\mathrm{TM}}$ testing. It is possible that the FFPE slices used for Idylla ${ }^{\mathrm{TM}}$ testing did not contain enough cells with instability for detection.

Five samples were also tested externally along with 21 other MSI samples, to determine whether another testing kit would give different results. The external testing was done using the Promega MSI Analysis System. This test uses seven biomarkers, five quasi monomorphic (BAT-25, BAT-26, MONO-27, NR-21 and NR-24) and two polymorphic markers (Penta C and Penta D). This analysis method needs two samples, namely a healthy tissue sample and the tumor sample. These are then compared for differences with the five monomorphic markers. If the microsatellites in the tumor tissue sample are different from that of the tumor in more than two biomarkers, then the sample is classified as MSI, and if one or none of the biomarkers are different from that of the normal sample, then the sample is classified as MSS. The two polymorphic markers are used as a control to ensure that the two samples being compared actually belong to the same patient. Since these markers are polymorphic, they have a high probability of corresponding with the sample despite being MSI-H.

Using this method, none of the samples showed significant instability. There is a difference in peak density in the tumor samples in comparison to the healthy samples. There was, however, no shift in the peaks, meaning, no instability present. This could be due to a lack of cancerous cells present in the tumor samples. Some of the samples analysed date back to 2007. It is possible that, over time, the FFPE blocks have been used and slices cut away from the tumor that only healthy tissue was left on the block.

By comparing both methods, it appeared that Idylla ${ }^{\mathrm{TM}}$ is only capable of detecting instability in CRC samples and not in samples from other origins. This is mainly due to marker specificity in the tumor environment and the fact that certain genes are more prone to mutations as a result of MSI than others. Overall, for MSI testing the Idylla ${ }^{\mathrm{TM}}$ system is reliable for colorectal samples to a certain degree. Considering only colorectal samples, with a specificity of $100 \%$ it can correctly identify MSS samples, but there is a chance that MSI samples are also identified as stable with an NPV of $88.8 \%$. The sensitivity, however, is lower at $85.7 \%$, but if the sample is identified as unstable, the likelihood that this is correct is $100 \%$ (PPV).

However, our study has some bias, which is the reason that we deem it to be a pilot study used to generate a hypothesis. First, the case number is rather low, which is results from the fact that the available funding was limited and further commercial MSI testing kits exceeded our grant budgets. Second, a general phenomenon in molecular pathology, the residual specimens' amount left for study purposes was limited after routine diagnostics were finished, thus we were not able to test all samples with all assays.

Future studies should systematically test tumors from different origin, metastases of those tumors, and make use of broad, empiric approaches including a wide spectrum of microsatellites in order to elucidate the phenomenon observed in the present study.

MSI/MSS testing may alternatively be performed by NGS protocols and may require additional analyses of the tumor mutational burden (TMB). However, these assays are not available in all laboratories, thus making the rapid detection methods such as the Idylla assay a rapid and broadly available tool for therapy relevant daily routine diagnostics.

\section{Conclusions and Hypothesis}

Taking into account these results and our previous study $[4,5]$ on the microsatellite instability in tonsillar tumors, we carefully hypothesize that microsatellite instability depends not only on the assay being used for testing, but may be tumor type specific. This leads to the conclusion that, to date, 
MSI testing may only be valid for a few tumor entities and that further systematic studies are required in order to evaluate if all (common) tumor types may have specific instability patterns and to which microsatellites are proper markers for the respective tumor types. A putative consequence for the current clinical and pathological practice could be that MSI testing algorithms established for distinct entities such as colorectal cancer may lead to false positive or, more likely, false negative results in another tumor entity unless specific patterns have been established.

Author Contributions: Conceptualization, O.S. and V.S.; methodology, T.H., C.S., R.-L.T., and M.P.; validation, O.S., M.B., and V.S.; formal analysis, M.B.; investigation, J.L.; writing-original draft preparation, O.S. and V.S.; writing - review and editing, V.S.; supervision, V.S.; funding acquisition, O.S. All authors have read and agreed to the published version of the manuscript.

Funding: This research was funded by the Beatrix Lichtken Stiftung and the Lörcher-Stiftung, both located in Cologne.

Conflicts of Interest: The authors declare no conflict of interest.

\section{References}

1. Murphy, K.M.; Zhang, S.; Geiger, T.; Hafez, M.J.; Bacher, J.; Berg, K.D.; Eshleman, J.R. Comparison of the Microsatellite Instability Analysis System and the Bethesda Panel for the Determination of Microsatellite Instability in Colorectal Cancers. J. Mol. Diagn. 2006, 8, 305-311. [CrossRef] [PubMed]

2. Nojadeh, J.N.; Sharif, S.B.; Sakhinia, E. Microsatellite instability in colorectal cancer. EXCLI J. 2018, 17, 159-168. [PubMed]

3. Van Cutsem, E.; Cervantes, A.; Adam, R.; Sobrero, A.; Van Krieken, J.H.; Aderka, D.; Aguilar, E.A.; Bardelli, A.; Benson, A.; Bodoky, G.; et al. ESMO consensus guidelines for the management of patients with metastatic colorectal cancer. Ann. Oncol. 2016, 27, 1386-1422. [CrossRef] [PubMed]

4. Förster, I.; Brockmann, M.; Schildgen, O.; Schildgen, V. Microsatellite instability testing in colorectal cancer using the QIAxcel Advanced advanced platform. BMC Cancer 2018, 18, 484. [CrossRef] [PubMed]

5. Höpken, M.; Förster, I.; Maune, S.; Brockmann, M.; Schildgen, O.; Schildgen, V. Association of the Human Bocavirus With Tonsil Squamous Cell Carcinomas. Front. Microbiol. 2018, 9, 2450. [CrossRef] [PubMed]

6. Schlotterer, C.; Ritter, R.; Harr, B.; Brem, G. High mutation rate of a long microsatellite allele in Drosophila melanogaster provides evidence for allele-specific mutation rates. Mol. Boil. Evol. 1998, 15, 1269-1274. [CrossRef] [PubMed]

7. Cortes-Ciriano, I.; Lee, S.; Park, W.-Y.; Kim, T.-M.; Park, P.J. A molecular portrait of microsatellite instability across multiple cancers. Nat. Commun. 2017, 8, 15180. [CrossRef] [PubMed]

8. Herman, J.G.; Umar, A.; Polyak, K.; Graff, J.R.; Ahuja, N.; Issa, J.-P.J.; Markowitz, S.; Willson, J.K.V.; Hamilton, S.R.; Kinzler, K.W.; et al. Incidence and functional consequences of hMLH1 promoter hypermethylation in colorectal carcinoma. Proc. Natl. Acad. Sci. USA 1998, 95, 6870-6875. [CrossRef] [PubMed]

9. Bonneville, R.; Krook, M.A.; Kautto, E.A.; Miya, J.; Wing, M.R.; Chen, H.-Z.; Reeser, J.W.; Yu, L.; Roychowdhury, S. Landscape of Microsatellite Instability Across 39 Cancer Types. JCO Precis. Oncol. 2017, 2017, 1-15. [CrossRef] [PubMed]

10. Schiemann, U.; Müller-Koch, Y.; Gross, M.; Daum, J.; Lohse, P.; Baretton, G.; Muders, M.; Mussack, T.; Kopp, R.; Holinski-Feder, E. Extended Microsatellite Analysis in Microsatellite Stable, MSH2 and MLH1 Mutation-Negative HNPCC Patients: Genetic Reclassification and Correlation with Clinical Features. Digestion 2004, 69, 166-176. [CrossRef] [PubMed]

(C) 2020 by the authors. Licensee MDPI, Basel, Switzerland. This article is an open access article distributed under the terms and conditions of the Creative Commons Attribution (CC BY) license (http://creativecommons.org/licenses/by/4.0/). 